\title{
Observation of Galactic Cosmic Rays and Gamma Rays with the High Altitude Water Cherenkov Observatory
}

\section{Segev BenZvi*广}

Department of Physics and Astronomy, University of Rochester, Rochester, NY, USA

E-mail: sybenzviepas.rochester.edu

\begin{abstract}
The High-Altitude Water Cherenkov Observatory, or HAWC, is carrying out an unbiased survey of cosmic rays and gamma rays from the Northern Hemisphere between $100 \mathrm{GeV}$ and 100 $\mathrm{TeV}$. HAWC is currently the only high-uptime wide-field $\mathrm{TeV}$ observatory in operation, and has a robust program to search for flares and other transient sources of gamma rays. The detector is also well suited to observe spatially extended regions of gamma-ray emission and cosmic-ray anisotropy. HAWC recently concluded its first year of data taking with the complete detector. The results include not only observations of many known $\mathrm{TeV}$ point sources, but also extended emission from Galactic objects like the Geminga supernova remnant. These results have implications for the origins of several astrophysical anomalies observed in the cosmic-ray data, such as the excess of Galactic positrons at Earth. We will describe results from HAWC with a focus on the observation of cosmic rays and Galactic sources of gamma rays.
\end{abstract}

38th International Conference on High Energy Physics

3-10 August 2016

Chicago, USA

\footnotetext{
* Speaker.

${ }^{\dagger}$ For the HAWC Collaboration.
} 


\section{Introduction}

The High Altitude Water Cherenkov (HAWC) Observatory is a gamma-ray and cosmic-ray air shower detector located 4100 meters above sea level at $19^{\circ} \mathrm{N}$ latitude in Sierra Negra, Mexico. The detector is operating continuously with $>90 \%$ uptime, including scheduled maintenance periods, and provides an unbiased wide field-of-view survey of the Northern Hemisphere.

HAWC is sensitive to primary cosmic rays between $100 \mathrm{GeV}$ and $100 \mathrm{TeV}$. At these energies the detector observes the spectrum of the cosmic rays in a transition region between satellite-based detectors such as AMS (which observe primary cosmic rays directly) and ground-based air shower detectors (which observe primary particles indirectly). HAWC records cosmic-ray showers at an event rate $>20 \mathrm{kHz}$, and the resulting high statistics provide sensitivity to the anisotropy in the arrival direction distribution of the cosmic rays at the $10^{-4}$ level [1]. HAWC can also observe the strong deficits (or "shadows") in the cosmic ray flux created by the Moon and Sun. Using the deflection of the shadows in the geomagnetic field, the observatory can discriminate between positively and negatively charged particles, and hence can estimate the fluxes of cosmic antiparticles $\left(\bar{p}, e^{+}\right.$, etc.) at energies above the limits of direct detectors [2].

Above $1 \mathrm{TeV}$ the information recorded by the detector can be used to effectively filter out cosmic-ray events. Between $1 \mathrm{TeV}$ and $100 \mathrm{TeV}$ HAWC is carrying out an unbiased wide fieldof-view survey of gamma rays from the Northern Hemisphere. The high uptime of HAWC allows for the observation of transient sources of gamma rays from $2 / 3$ of the sky each day $[3,4,5]$. Its high energy reach enables HAWC to distinguish gamma rays originating in $\pi^{0}$ decay from inverse Compton scattered photons, allowing us to search for accelerators of hadronic cosmic rays in the Milky Way. Finally, thanks to the 2 sr instantaneous field of view of the array, HAWC is sensitive to gamma rays from very spatially extended galactic and extragalactic sources [6]. These sources include diffuse emission, which is important for the identification of the origin of cosmic neutrinos as well as the characterization of gamma rays from Dark Matter annihilation and decay.

Construction of HAWC ended in March 2015. In this proceeding we present preliminary results from the first 15 months of data taking with HAWC, and discuss prospects for the future.

\section{HAWC Operation and Analysis}

The HAWC Observatory comprises 300 water Cherenkov detectors (WCDs) covering an area of $20,000 \mathrm{~m}^{2}$ [7]. The layout of the WCDs is illustrated in Fig. 1. Each WCD is a cylindrical $5 \mathrm{~m} \times 7.3 \mathrm{~m}$ steel tank. Its interior is lined with a plastic bladder and filled with 200,000 L of purified water. Four photomultipliers anchored to the bottom of the WCD record the Cherenkov light produced when charged particles in an air shower pass through the detector.

The relative timing of charged particle hits in the WCDs is used to reconstruct the arrival direction of the primary particle which initiated the air shower, and the number of triggered channels in the detector is roughly proportional to the energy of the primary cosmic ray or gamma ray. The accuracy of the directional reconstruction ranges from about $1^{\circ}$ below $1 \mathrm{TeV}$ to $0.1^{\circ}$ above $10 \mathrm{TeV}$.

As shown in Fig. 1, cosmic-ray showers are characterized by "clumpy" deposits of charge at large distances from the core of the air shower, while gamma rays appear to have a relatively low variance in the charge as a function of distance from the shower core. Cuts on the spatial distribu- 

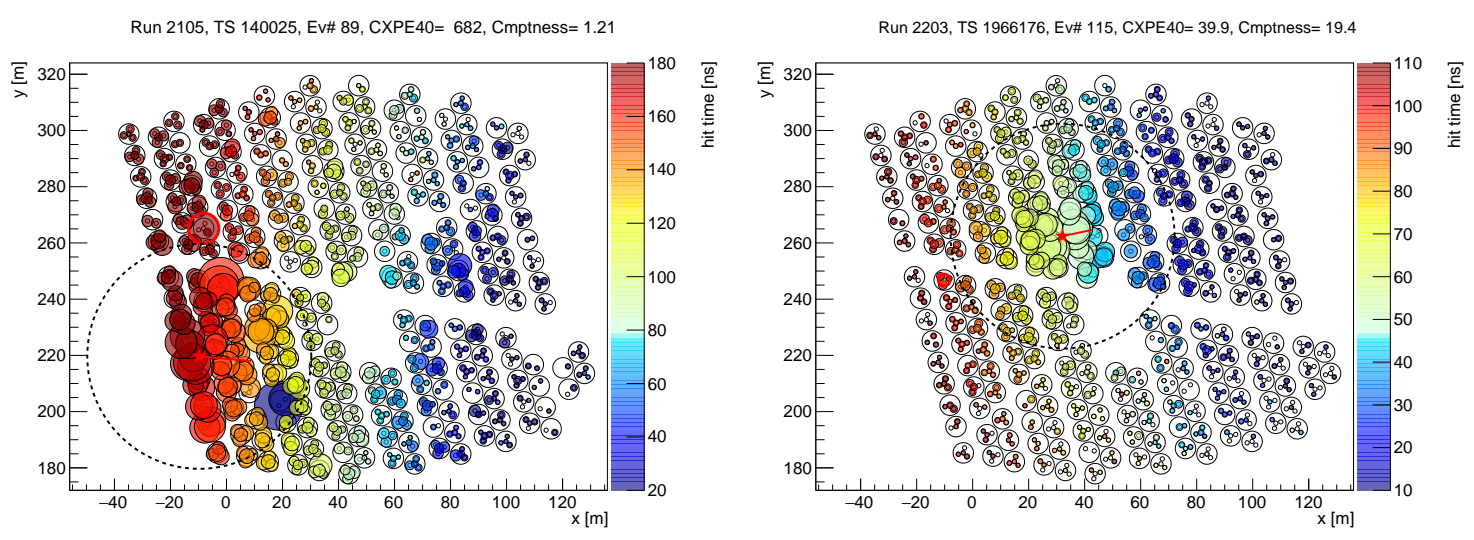

Figure 1: Left: event display showing a cosmic-ray shower triggering the complete HAWC array. The colors indicate the trigger times of PMTs in the detector, and the circle area is proportional to the number of photoelectrons recorded by each PMT. Small empty circles indicate untriggered PMTs. Right: a gamma-ray event.

tion of charge recorded in each trigger are used to filter out cosmic-ray events and keep gamma-ray showers. The efficiency of the cosmic-ray cuts improves as a function of energy, ranging from about $5 \%$ at $1 \mathrm{TeV}$ to $0.1 \%$ at $10 \mathrm{TeV}$, while the gamma ray passing efficiency is about $75 \%$.

The search for gamma-ray sources is carried out using a binned analysis of fine spatial pixels and coarse shower size bins using the fraction of active PMTs triggered. The cosmic-ray background filter and detector point spread function are optimized in each shower size bin. Spatial and spectral models of gamma-ray sources are then forward-folded through the detector response using simulated events and fit to data in the shower size bins. The fit is performed using a maximum likelihood

$$
\ln \mathscr{L}(\vec{n} \mid \vec{\theta})=\sum_{i=1}^{N_{\text {bin }}} \sum_{j=1}^{N_{\text {pix }}} n_{i j} \ln \lambda_{i j}(\vec{\theta})-\lambda_{i j}(\vec{\theta})-\ln n_{i j} !
$$

where the observed counts $n$ are compared to the model counts $\lambda$, which are the sum of background and signal contributions: $\lambda_{k}=B_{k}+\sum_{l} f_{k l}(\vec{\theta})$. In principle several sources can contribute to the model counts in each pixel, and the source spatial and spectral parameters $\vec{\theta}$ are estimated by maximizing $\ln \mathscr{L}$. To estimate the significance of a detection, we use the test statistic defined by the likelihood ratio of signal plus background versus background alone:

$$
\mathrm{TS}=2 \Delta \ln \mathscr{L}
$$

The significance of the detection is given by $\sqrt{\mathrm{TS}}$.

\section{Survey of the Inner Galaxy}

Figure 2 shows the region of the inner Galaxy visible to HAWC from its location in central Mexico. The skymap shows $\sqrt{\mathrm{TS}}$ as a function of galactic longitude going from the Galactic Center region (bottom right) to the Cygnus region (top left), one of the largest stellar nurseries in the Milky Way. The green labels in the skymap indicate sources discovered by previous $\mathrm{TeV}$ surveys in the 
Northern and Southern Hemispheres, while the yellow labels indicate local maxima in $\sqrt{\mathrm{TS}}$ that were identified as gamma-ray source candidates in 15 months of HAWC data. Due to the statistical penalty incurred by a blind search of this region, $\sqrt{\mathrm{TS}} \approx 7$ corresponds to a $5 \sigma$ detection after trials.
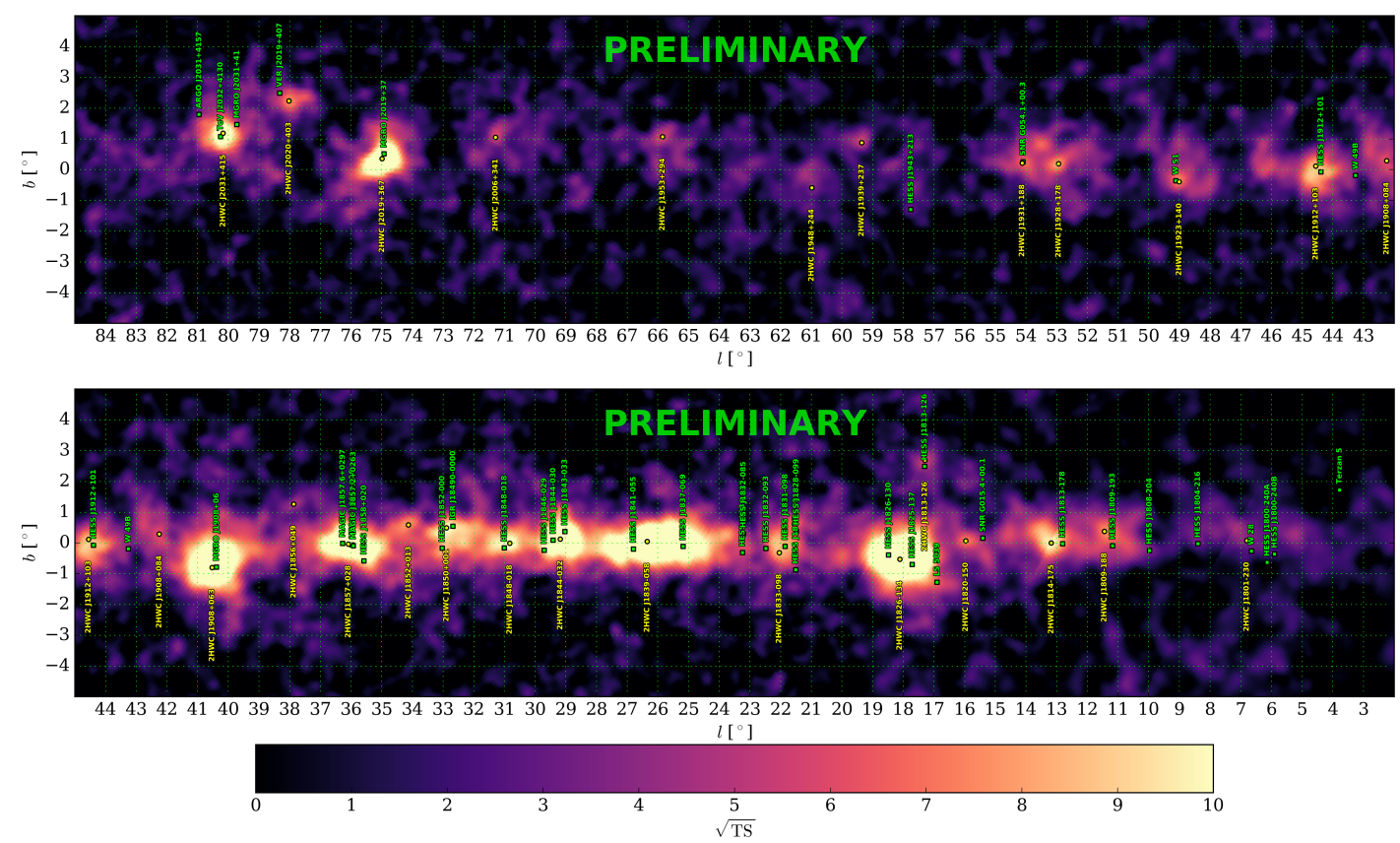

Figure 2: Sources of gamma rays between 1 and $100 \mathrm{TeV}$ along the region of the Galactic Plane observed by HAWC.

Several new source candidates with no TeV counterparts have been observed in this data set, and these discoveries are currently being followed up at other wavelengths. One such region of interest is shown in Fig. 3. The region contains one previously known TeV source, a nearby supernova remnant with an energetic pulsar, and two new sources discovered by HAWC. The detection of the new sources in a region previously explored by $\mathrm{TeV}$ detectors exhibits the power of the HAWC Observatory; due to its wide field of view it can cover many regions of the sky without dedicated observing time. In addition, while HAWC is not as sensitive to point sources of gamma rays as other $\mathrm{TeV}$ observatories, it is less affected by systematic uncertainties in the sky background that can prevent the detection of point sources.

\section{Very Extended Emission: Geminga and PSR B0656+14}

Among the new sources of gamma rays detected by HAWC are two very spatially extended regions of emission in the outer Galaxy: Geminga and PSR B0656+14. Both objects are pulsar wind nebulae located $\sim 250 \mathrm{pc}$ from Earth. Neither object has been observed previously at TeV, though the Milagro Collaboration reported a sub-5 $\sigma$ detection of extended emission from Geminga in 2009 [8]. While the Geminga pulsar has been observed in X-rays and $\mathrm{GeV}$ gamma rays [9, 10], the observations with HAWC are the first time extended emission has been observed from its nebula. The lack of multi-wavelength observations of the nebula is not currently understood. 


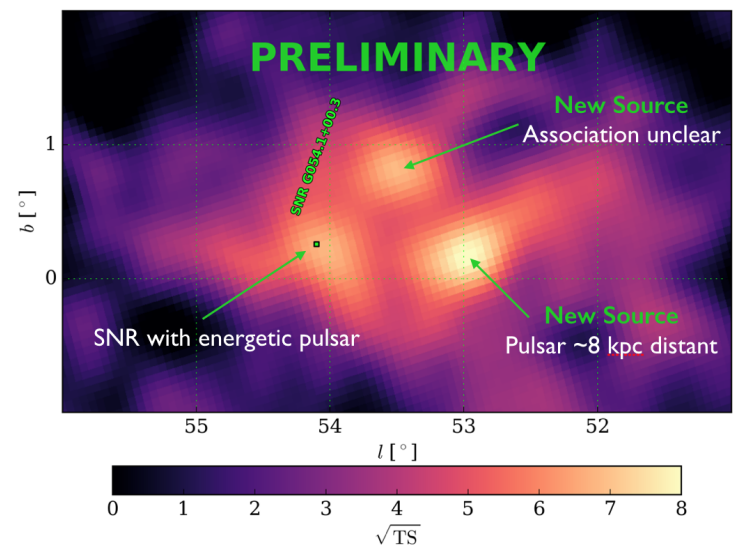

Figure 3: Zoom-in of a region of the inner Galaxy showing two previously unknown point sources of gamma rays.
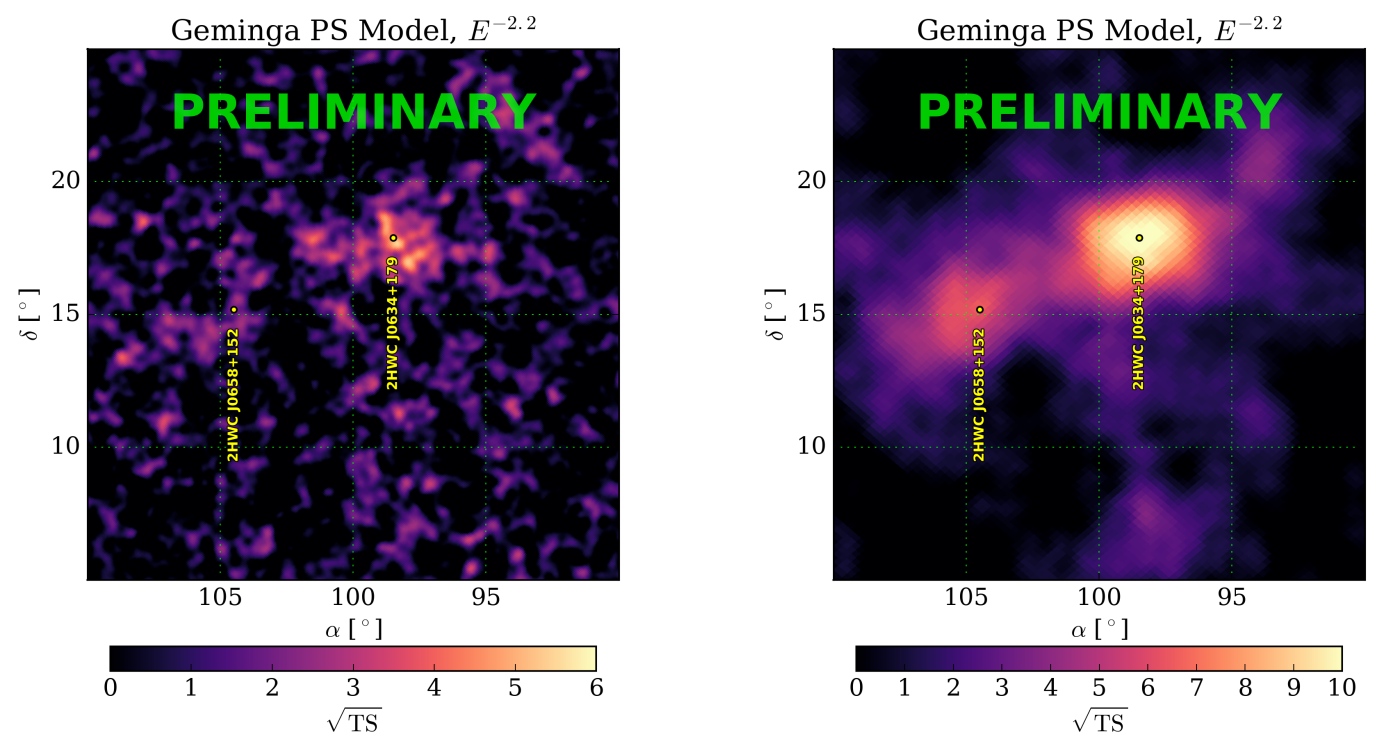

Figure 4: Spatially extended regions of emission from Geminga (2HWC J0634+179) and PSR B0656+14 (2HWC J0658+152), shown in equatorial coordinates. The left plot shows the region after smoothing by the detector point spread function; the right plot shows the same region smoothed by a $2^{\circ}$ top hat function.

Due to their proximity to the Solar system, Geminga and PSR B0656+14 are strong candidates for the source of the local electron and positron flux observed at Earth. These objects may also be the source of the local excess of positrons above the rate expected from cosmic ray interactions in the interstellar medium. Since the positron excess is also a signature of dark matter annihilation, the association of the excess with nearby particle accelerators is of considerable interest. We are currently using the observations with HAWC to investigate this possibility. 


\section{Conclusions}

HAWC has been operating continuously since March 2015 and has accumulated one of the world's largest data sets of cosmic rays and gamma rays above $1 \mathrm{TeV}$. Among the first observations are the detection of $>30$ point sources in the inner Galaxy, which includes 9 objects not previously detected at $\mathrm{TeV}$. Very extended emission has also been observed from the nebulae surrounding Geminga and PSR B0656+14, and we are exploring the possibility that these nearby objects contribute significantly to the local $e^{+} e^{-}$flux at Earth.

HAWC is currently funded to operate through 2020. The HAWC collaboration is now constructing a high-energy "outrigger" extension of the central array, which will increase the effective area to gamma rays above $10 \mathrm{TeV}$ by up to a factor of four. We expect the high-energy extension to be in operation in 2017.

\section{Acknowledgments}

This work was supported by the Department of Energy Office of High Energy Physics under grant number DE-SC0008475.

\section{References}

[1] HAWC Collaboration, A. U. Abeysekara et al., Observation of Small-scale Anisotropy in the Arrival Direction Distribution of TeV Cosmic Rays with HAWC, Astrophys. J. 796 (2014), no. 2 108, [arXiv:1408.4805].

[2] HAWC Collaboration, S. BenZvi, D. Fiorino, Z. Hampel-Arias, and M. U. Nisa, Towards a Measurement of the $e^{+} e^{-}$Flux above 1 TeV with HAWC, PoS ICRC2015 (2016) 248, [arXiv:1508.0346].

[3] HAWC Collaboration, R. J. Lauer and P. W. Younk, Results from monitoring TeV blazars with HAWC, PoS ICRC2015 (2016) 716, [arXiv: 1508 . 0447 ].

[4] HAWC Collaboration, J. Wood, All-sky sensitivity of HAWC to Gamma-Ray Bursts, PoS ICRC2015 (2016) 784, [arXiv:1508.0412].

[5] HAWC Collaboration, S. BenZvi, Looking for Gamma-ray Emission from TeV Binary Candidates with HAWC, PoS ICRC2015 (2016) 714, [arXiv: 1508 . 0358].

[6] HAWC Collaboration, B. M. Baughman and J. Wood, TeV Gamma-Ray Emission Observed from Geminga with HAWC, in Proceedings, 34th International Cosmic Ray Conference (ICRC 2015): The Hague, The Netherlands, July 30-August 6, 2015, 2015. arXiv:1508.0349.

[7] HAWC Collaboration, A. J. Smith, HAWC: Design, Operation, Reconstruction and Analysis, PoS ICRC2015 (2016) 966, [arXiv: 1508 . 0582].

[8] A. A. Abdo et al., Milagro Observations of TeV Emission from Galactic Sources in the Fermi Bright Source List, Astrophys. J. 700 (2009) L127-L131, [arXiv: 0904 . 1018].

[9] P. A. Caraveo, G. F. Bignami, A. DeLuca, S. Mereghetti, A. Pellizzoni, R. Mignani, A. Tur, and W. Becker, Geminga's tails: a pulsar bow shock probing the interstellar medium, Science 301 (2003) 1345-1348.

[10] Fermi-LAT Collaboration, A. A. Abdo et al., Fermi LAT observations of the Geminga pulsar, Astrophys. J. 720 (2010) 272-283, [arXiv: 1007.1142$].$ 